\title{
Modelos predictivos de dispersión de Hypothenemus hampei (Coleoptera: Curculionidae) en cafetales con diferentes usos de suelo adyacente en Turrialba Costa Rica
}

\author{
Predictive dispersal models of Hypothenemus hampei (Coleoptera: Curculionidae) \\ in coffee plantations with different uses of adjacent soil in Turrialba, Costa Rica
}

\author{
Karime Montes Escobar a, Carlos Salas Macías ${ }^{\mathrm{b} *}$, Guillermo Rogel Zambrano ${ }^{\mathrm{c}}$ \\ ${ }^{a}$ CATIE, Maestría en Agroforestería Tropical, Turrialba, Costa Rica. \\ *Autor de correspondencia: ${ }^{\text {b }}$ Universidad Técnica de Manabí, Facultad de Ingeniería Agronómica, \\ Lodana, Santa Ana, Ecuador, carlos.a.salas.m@gmail.com \\ c Universidad Nacional Agraria La Molina, Maestría en Entomología, Lima, Perú.
}

\begin{abstract}
SUMMARY
Statistical models were developed that describe the dispersion of the borer (Hypothenemus hampei) in land uses adjacent to coffee plantations (grass, cane and forest). Sampling was conducted with a methodology of transects in farms located in the canton of Turrialba, within the Central Volcanic Corridor Talamanca (CBVCT) in Costa Rica. In each transect BrocapTM traps were placed every $10 \mathrm{~m}$, capturing the berry borer for six months. With the purpose of studying the distance at which the insect is dispersed in different adjacent land uses, three statistical modeling approaches were used 1) non-linear models, 2) general linear mixed models and 3) generalized linear mixed models. These different modeling approaches within each model type were evaluated with the information criteria AIC and BIC. To compare these different modeling approaches, the Pearson correlation coefficient between the predicted values for each model and the observed values was used. The results of this work suggest that the best fit for the description of the insect dispersion was according to the generalized linear mixed model. Based on this model, the approximate maximum distance of dispersal occurred in the adjacent sugar cane land use at $30 \mathrm{~m}$ and the minimum distance was in the adjacent forest land use at $10 \mathrm{~m}$, suggesting that the cane cultivation serves as a transit for the coffee borer to move between the coffee plantations, and the soil use that best controls the movement of the coffee borer is the forest.
\end{abstract}

Key words: Statistical models, ecosystem services, insect dispersion, insect abundance.

RESUMEN

Mediante datos obtenidos en una investigación previa, se ajustaron modelos estadísticos que describen la dispersión de la broca (Hypothenemus hampei) en usos adyacentes a los cafetales (pasto, caña y bosque). Los muestreos se realizaron en fincas del cantón Turrialba, Costa Rica. En cada transecto se dispusieron trampas Brocap cada 10 m, registrándose la captura de broca durante el periodo comprendido entre febrero y julio de 2009. A fin de estudiar la distancia a la que se dispersa el insecto en los distintos usos de suelo adyacentes, se usaron diferentes aproximaciones de modelación estadística: 1) modelos no-lineales, 2) modelos lineales generales mixtos y 3) modelos lineales generalizados mixtos. Las distintas aproximaciones de modelación dentro de cada tipo de modelo fueron evaluadas con los criterios de información AIC y BIC. Para comparar las distintas aproximaciones de modelación, se usó como criterio el coeficiente de correlación de Pearson entre los valores predichos por cada modelo y los valores observados. Los resultados sugieren que el mejor ajuste para describir la dispersión de la broca en los usos adyacentes fue el modelo lineal generalizado mixto. Según este modelo la distancia máxima de dispersión fue en el uso de suelo caña a los 30 m y la mínima distancia fue en el uso de suelo adyacente bosque a los $10 \mathrm{~m}$, sugiriendo que el cultivo de caña sirve como tránsito para que la broca se mueva entre los cafetales, y el uso de suelo que mejor controla el movimiento de la broca es el bosque.

Palabras clave: modelos estadísticos, dispersión de insectos, abundancia de insectos, usos de suelo.

\section{INTRODUCCIÓN}

Se considera al café (Coffea arabica L.) como uno de los cultivos de gran importancia a nivel mundial, sobre todo en los países productores, en donde muchas veces no solo representa un rubro de relevancia económica, sino que además engloba aspectos socio-culturales. Su origen se ubica en Etiopía, donde se encuentra en los sotobosques por encima de los $1.500 \mathrm{~m}$ de altitud. A pesar de su extensa difusión en la franja intertropical, los países 
de mayor producción se encuentran en América Latina y el Caribe.

Sin lugar a dudas el desarrollo de la caficultura en los países productores significó un motor de crecimiento y fuente de divisas, no obstante, a finales del siglo XX, este rubro atravesó una de las peores crisis de la historia debido al exceso de la oferta en el mercado mundial, lo que consecuentemente ocasionó la caída de los precios y que muchos de los productores fragmenten sus cafetales o simplemente los remplace por otros cultivos (Rodríguez 2014).

De la mano de los inconvenientes de mercado, es innegable la lucha a la que se enfrentan los productores de café con uno de sus principales enemigos de este cultivo, la broca Hypothenemus hampei (Ferrari) (Coleoptera: Curculionidae) (Rebolledo et al. 2016). Este insecto originario de África ocasiona daños importantes en los granos, en donde realiza galerías y oviposita, permaneciendo en el interior mientras su progenie se desarrolla.

El mayor daño se debe a que la broca no solo perfora los frutos tiernos, sino también los granos maduros, produce daño fisiológico, pudrición de frutos y la caída de estos al suelo (Jaramillo et al. 2015), provocando pérdidas directas en el peso y afectando seriamente la productividad (Guharay et al. 2000) y calidad del mismo.

Pese al impacto que $H$. hampei tiene sobre los cultivos de $C$. arabica, existen evidencias que permiten pensar que el hospedero original fue Coffea canephora Pierre Ex. A. Froehner, la cual se desarrolla a alturas menores a $1.500 \mathrm{~m}$ s.n.m., donde se presentan las condiciones óptimas para el desarrollo de la plaga (Guharay et al. 2000). Actualmente, la broca se encuentra en todas las regiones del mundo donde hay cultivos de café. Esta colonización se ha dado mediante el transporte de semillas infectadas en sacos, contenedores y barcos.

En este sentido, existen innumerables factores que podrían ayudar a la dispersión de los cuales se podría citar al traslado de granos infectados al acopio, el uso de instrumentos infectados, la migración de trabajadores que concurren en las labores de recolección de café, la adherencia de las brocas en la vestimenta de recolectores de café (corteros), los sacos o costales donde se traslada el fruto cortado y el uso de pulpa sin fermentar como abono en las fincas, entre otros.

Pese a lo expuesto y a fin de evitar la dispersión de la broca hacia cafetales circundantes, se han desarrollado múltiples investigaciones que han permitido conocer el movimiento del agente en condiciones de campo, proponiendo medidas para disminuir las poblaciones. No obstante, varias de las tecnologías incrementan los costos de producción, de tal manera los productores optan por utilizar plaguicidas cuyo uso inadecuadamente en algunos casos podrían provocar la aparición de plagas secundarias, elevando nuevamente los costos y produciendo daños más graves (Guharay et al. 2000).

Frente a esta situación, es importante reconocer no solo el efecto de la interacción del ambiente, el hospedero, la plaga, sino también aspectos biológicos y ecológicos de las plagas como sus mecanismos de reproducción y su habilidad para moverse a través del paisaje (Olivas 2010). En el caso de la broca, el mecanismo de dispersión es una limitante ya que el insecto para colonizar nuevas áreas, en ocasiones, debe volar largas distancias (Baker 1984). La dirección y la distancia de vuelo están relacionadas entre otros aspectos con las condiciones geográficas del sitio en donde esté ubicado el cafetal, los factores climáticos y la dirección de los vientos (Damon 2000).

El presente trabajo tiene como base conceptual el hecho de que, conociendo la relación existente entre el paisaje, la dinámica del insecto y los efectos de suelo adyacentes sobre las poblaciones de $H$. hampei, a nivel agronómico se podrán proponer configuraciones de paisaje que actúen como barreras para su dispersión. Bajo estas consideraciones, se propone como objetivos: 1) determinar un modelo predictivo que describa mejor el patrón de dispersión de la broca hacia fuera de los cafetales con diferentes usos de suelo adyacente; 2) identificar el uso de suelo adyacente que limita la dispersión de la broca hacia fuera de los cafetales.

\section{MÉTODOS}

Área de estudio. La data utilizada para este trabajo fue recopilada por Olivas (2010) en el Corredor Biológico Volcánica Central Talamanca (CBVCT). Los cafetales (C. arabica) en estudio tuvieron usos adyacentes similares, siendo predominantes y comunes en ellos los cultivos de caña, pasto y bosque (figura 1). Dichos sitios de muestreo fueron las fincas: a) finca Maquina Vieja, b) finca CATIE, c) finca Colima, d) finca El volcán, e) finca More Costa Rica, f) finca El Romeral (más información sobre las características agronómicas de los sitios en Olivas 2010).

La metodología utilizada por Olivas (2010) para recabar los datos de dispersión se cita a continuación:

Se establecieron trampas BROCAP ${ }^{\circledR}$ Dufour (Dufour 2007) y atrayentes, con el fin de conocer la cantidad de broca que emigra del café hacia usos de suelo adyacentes y la forma en que esta se dispersa. Su establecimiento estuvo supeditada a la cosecha del café, ya que la mayor dispersión de la broca se muestra en periodos de pos cosecha (Dufour 2007).

Se ubicaron tres transectos de $180 \mathrm{~m}$ de largo en cada una de las combinaciones de usos (café-caña, café-bosque y café pasto). Entre cada transecto la distancia varió entre 30 y $40 \mathrm{~m}$. Estas distancias entre transectos garantizan la independencia entre las observaciones, ya que los difusores o atrayentes tienen un radio de acción de 12,5 m.

Se instalaron 18 trampas por transecto, cada una a $10 \mathrm{~m}$ de distancia a una altura aproximada de 1,2 m entre el difusor y el suelo, ubicando cuatro trampas en las áreas de café, hasta $30 \mathrm{~m}$ del cafetal. Los 14 restantes, se dispusieron a lo largo del transecto en los usos adyacentes (caña, pasto o bosque). Se situaron 54 trampas por combinación y 108 por finca, para un total de 648 en las seis fincas. Cada mes se hicieron tres colecciones, con un total de 12 a 15 


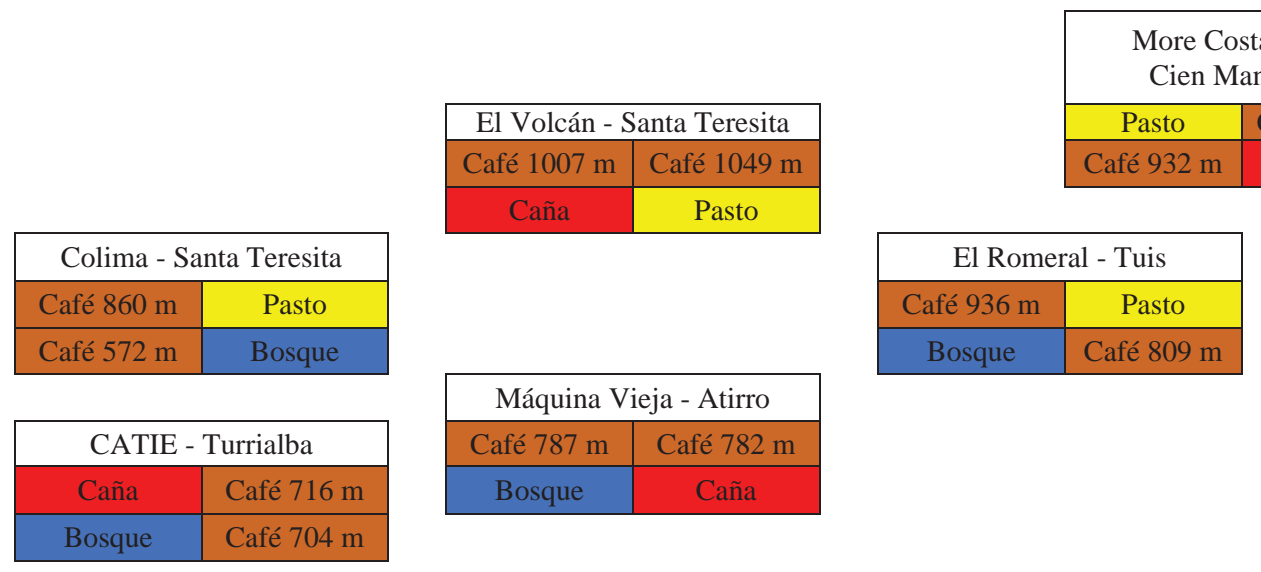

Figura 1. Representación de las parcelas de café seleccionadas por Olivas (2010), la altitud y los usos de suelo colindante, situadas en el cantón de Turrialba, Costa Rica 2009.

Representation of the coffee plots selected by Olivas (2010), altitude and adjacent land uses, located in Turrialba, Costa Rica 2009.

muestreos por finca durante seis meses. Los conteos de la broca se hicieron de forma manual, teniendo en cuenta que ninguna trampa capturo más de 1.000 individuos.

Análisis estadístico. Con el fin de definir el mecanismo de dispersión de la broca a lo largo del paisaje, el total de la captura de broca que se obtuvo del borde del cafetal hacia los usos de suelo adyacentes en el periodo de estudio de Olivas (2010) fue usado como variable respuesta. Los conteos fueron relacionados con las distancias mediante gráficos de dispersión y estadística no paramétrica.

Estas gráficas permitieron encontrar la función estadística aproximada que se ajusta mejor a los datos de la dispersión de la broca bajo las condiciones del estudio. Se usaron diferentes aproximaciones para encontrar la distancia de la dispersión de la broca dentro de los diferentes tipos de uso: 1) modelos no-lineales, 2) modelos lineales generalizados mixtos, 3) modelos lineales generales mixtos, 4) modelos de regresión por pasos (broken stick, vara quebrada).

Para evaluar el movimiento de la broca del cafetal a los distintos usos de suelo, se ajustaron cinco modelos no lineales que según Kot et al. (1996) son los más recomendados para ajustar curvas de dispersión y sirven para hacer pronósticos sobre el movimiento de los fenómenos, en este caso, la dispersión de la broca. Estos modelos se ajustaron para cada uso de suelo y en cada finca (cuadro 1).

Para la selección del mejor modelo se realizaron regresiones no lineales teniendo en cuenta el número total de brocas capturadas en función de las distancias a las que se encuentran fuera del café y dentro de los diferentes usos de suelo. Se usaron los criterios de información de Akaike (AIC) y de información bayesiana (BIC), que sirven para selección el modelo más parsimonioso (la preferencia por la explicación más simple entre varias alternativas).

Posteriormente, se analizaron las estimaciones de los parámetros de los modelos seleccionados, su error están- dar y sus límites de confianza del $(95 \%)$. Con esto se determinó el mejor modelo de ajuste que fue usado con el fin de encontrar las similitudes entre cada uso de suelo.

Para los diferentes usos de suelo, teniendo en cuenta los efectos de finca, se calcularon las distancias de dispersión de la broca. Para esto, luego de seleccionar el modelo que mejor ajustó, se igualó el modelo a cero (indicando que ya la broca no se dispersa) antes de despejar la distancia.

Otra aproximación considerada para encontrar la distancia a la que la broca no se dispersa en cada uso de suelo consistió en el uso de modelos lineales generales y mixtos (cuadro 2). Estos permitieron modelar la dispersión de broca que se encuentra en el borde del cafetal hacia los diferentes usos de suelo en función a las distancias que se encuentran dentro de las parcelas en estudio, cuyos efectos pueden considerarse tanto fijos como aleatorios.

$\mathrm{Al}$ analizar los datos, primero se ajustaron modelos con distintas estructuras de covarianza, combinando estructuras de correlación residual (diferentes estructuras de correlación de los errores), heteroscedasticidad residual (permi-

Cuadro 1. Modelos no lineales utilizados en la estimación de la relación foco de dispersión y cada clase de distancia en la que fueron colocadas las trampas.

Nonlinear models used in the estimation of the dispersion focus ratio and each distance class in which the traps were placed.

\begin{tabular}{ccc}
\hline Modelos & Función & Familia \\
\hline 1 & $e^{\left(a-b x^{2}\right)}$ & Exponencial \\
2 & $a-b \ln x$ & Logarítmico \\
3 & $a-b \ln x+\frac{c}{x}$ & Logarítmico \\
4 & $e^{(a-b \sqrt{x})}$ & Exponencial \\
5 & $e^{(a-b x)}$ & Exponencial \\
\hline
\end{tabular}


Cuadro 2. Modelos generalizados lineales y mixtos para comparar la estimación de la relación foco de dispersión y cada clase de distancia en la que fueron colocadas las trampas.

Generalized Linear and Mixed Models to compare the estimate of the dispersion focus ratio and each distance class in which the traps were placed.

\begin{tabular}{|c|c|c|c|c|c|c|c|}
\hline Modelo & Cov & E.A & Corr & coor $X$ & Crit.Agrup & Heter & Crit.Agrup \\
\hline 1 & - & - & - & - & - & - & - \\
\hline 2 & - & Sujeto & - & - & - & - & - \\
\hline 3 & - & - & corExp & distancia & sujeto & - & - \\
\hline 4 & - & Sujeto & corExp & distancia & sujeto & - & - \\
\hline 5 & - & Sujeto & corGaus & distancia & sujeto & - & - \\
\hline 6 & - & Sujeto & corLin & distancia & sujeto & - & - \\
\hline 7 & - & Sujeto & corSpher & distancia & sujeto & - & - \\
\hline 8 & - & - & - & - & - & VarIdent & Var \\
\hline 9 & - & - & - & - & - & VarIdent & distancia \\
\hline 10 & - & - & - & - & - & VarExp & - \\
\hline 11 & - & Sujeto & corExp & distancia & sujeto & VarIdent & distancia \\
\hline 12 & - & Sujeto & corExp & distancia & sujeto & varIdent & Var3 \\
\hline 13 & - & Sujeto & corExp & distancia & sujeto & varIdent & Tipo de uso*distancia \\
\hline 14 & - & Sujeto & corExp & distancia & sujeto & VarIdent & var3*tipo de uso \\
\hline 15 & - & - & corExp & distancia & sujeto & VarIdent & var3*tipo de uso \\
\hline 16 & P_cafe & Sujeto & corExp & distancia & sujeto & VarIdent & var3*tipo de uso \\
\hline
\end{tabular}

Donde: Cov: estructura de covarianza, E.A: Efecto aleatorio, Corr: Correlación de los errores, CoorX: Coordenada X, Crit.Agrup: Criterio de agrupamiento, Heter: heteroscedasticidad residual. corExp: Correlación espacial exponencial, Cor Gaus: correlación espacial Gaussiana, corLin: correlación espacial Lineal, CorSpher: correlación espacial esférica, varIdent: varianzas heterogéneas.

te seleccionar distintos modelos para la función de varianza) y efectos aleatorios. Teniendo en cuenta los criterios de información de Akaike (AIC) y el criterio de información Bayesiana (BIC), se eligió el modelo que mejor describió los datos, y con ese modelo se hicieron inferencias acerca de las medias.

Para encontrar el mejor modelo primero se comenzó por uno sencillo con pocos parámetros a estimar, y después se fueron adicionando parámetros hasta llegar a un modelo que contemplara las estructuras de los datos sin que deje de ser parsimonioso. En todos los casos se usó el mismo modelo de medias, ya que los factores de interés no cambiaron.

Para el primer modelo solo se declaran como efectos fijos a tipo de uso, distancia y la interacción tipo de uso $x$ distancia. El segundo modelo tiene la misma estructura que el primero, sin embargo, se adiciona a sujeto como efecto aleatorio.

El tercer modelo tiene la misma estructura del primer modelo, en este caso, no se declara sujeto como efecto aleatorio, pero se declara como función de correlación de los errores a corExp (Correlación espacial exponencial) con coordenada $\mathrm{X}$ a distancia y como criterio de agrupamiento a sujeto. En el cuarto modelo se declara nuevamente a sujeto como efecto aleatorio, con función de correla- ción corExp, con coordenada $\mathrm{X}$ a distancia y como criterio de agrupamiento a sujeto. El quinto modelo tiene la misma estructura del cuarto modelo, sin embargo, la función de correlación de los errores es cambiada por corGaus (correlación espacial Gaussiana).

El sexto modelo tiene la misma estructura que el cuarto modelo, sin embargo, la función de correlación de los errores es cambiada por corLin (correlación espacial Lineal). El séptimo modelo tiene la misma estructura del cuarto modelo, pero en este la función de correlación de los errores es cambiada por corSpher (correlación espacial esférica).

En el octavo modelo no se declara efecto aleatorio ni función de correlación de los errores, sin embargo, se declara varianzas distintas (varIdent) usando como criterio de agrupamiento a una variable que representa las varianzas de los datos en dos grupos en función de la distancia (0 representa la distancia cero y 1 representa al resto de las distancias). Esta variable sirve para declarar el hecho de que las varianzas son mucho mayores en el borde del cafetal (cercanas a la distancia cero), ya que los conteos son mayores, y caen drásticamente en el resto de las distancias. El agregado de este tipo de variables indicadoras se usa para contemplar heterogeneidad de varianzas usando un modelo más parsimonioso. 
El modelo nueve tiene la misma estructura que el octavo modelo, sin embargo, las varianzas heterogéneas (varIdent) cambian según las distancias. El modelo diez tiene la misma estructura que el octavo modelo, sin embargo, las varianzas heterogéneas (varIdent) es cambiada por varExp (función de varianza exponencial).

El modelo once tiene la misma estructura del modelo cuatro, sin embargo, se declara las varianzas heterogéneas (varIdent) según las distancias. El modelo doce tiene la misma estructura del modelo once, sin embargo, se reemplaza la varianza heterogénea (varIdent) usando como criterio de agrupamiento a una variable que representa las varianzas de los datos en tres grupos en función de la distancia (0 representa la distancia cero, 10 representa a la distancia 10 y 20 al resto de las distancias).

El modelo trece tiene la misma estructura que el modelo once, sin embargo, se reemplaza la varianza heterogénea (varIdent) usando como criterio de agrupamiento a Tipo de uso $\times$ distancia. El modelo catorce tiene la misma estructura que el modelo once, sin embargo, se reemplaza la varianza heterogénea (varIdent) usando como criterio de agrupamiento a var3×tipo de uso. El modelo quince tiene la misma estructura que el modelo catorce, sin embargo, no se declara efectos aleatorios. El modelo dieciséis tiene la misma estructura del modelo catorce, sin embargo, se declara la Covariable P_café dentro de los efectos fijos.

Posteriormente, se realizaron nuevos análisis estadísticos con modelos lineales generales y mixtos, transformando la variable respuesta conteo a logaritmo natural. Esto permitió modelar la cantidad de broca que se encuentra en el borde del cafetal hacia los diferentes usos de suelo en función a las distancias que se encuentran dentro de las parcelas en estudio, cuyos efectos pueden considerarse tanto fijos como aleatorios. Los modelos tienen la misma estructura que los modelos mencionados anteriormente, sin embargo, cambia la variable respuesta conteo por una transformación de ésta a logaritmo natural.

Por otra parte, para poder ver la relación del tipo de cultivo y las distancias sin el sesgo dado por el efecto de finca dentro de cada localidad, se utilizaron modelos lineales generalizados mixtos (cuadro 3); estos no solo permiten descontar el efecto de finca dentro de cada localidad sino también permiten contemplar diferentes distribuciones para el término de error. Estos modelos permiten ajustar directamente la variable número de insectos con una distribución Poisson, asumiendo entre otras cosas, que se incrementa la varianza al aumentar la media. Para este modelo se utilizó la función de enlace Log.

Estos modelos fueron analizados con diferentes estructuras, el primero tiene como variable independiente el conteo, como criterios de clasificación al tipo de uso y la distancia, declarando en los efectos fijos al tipo de uso, la distancia y la interacción tipo de uso×distancia. El segundo modelo presenta la misma estructura que el primero, pero se adiciona dentro de los criterios de clasificación y como efecto fijo la covariable cantidad de broca que se encuentra dentro del café en las parcelas adyacentes a cada tipo de uso considerado (P_café).

El tercer modelo tiene la misma estructura del segundo

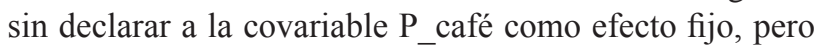
se declara como efecto aleatorio a los transectos dentro de cada finca, y uso (comúnmente llamado factor "sujeto", que indica el conjunto de unidades muestrales correlacionadas). Esta última variable fue declarada así, para tener en cuenta la estructura correlacionada asociada a las distancias dentro de un transecto.

El cuarto modelo, tiene la misma estructura que el primer modelo, agregándole como efecto fijo a $\mathrm{P}$ café $\mathrm{y}$ como efecto aleatorio a sujeto. Estos modelos también fueron analizados con los criterios de información AIC y BIC.

Finalmente, se realizaron análisis con modelos de regresión por pasos (broken stick, vara quebrada) con diferentes alternativas de expresión de las rectas (cuadro 4). La intersección de las dos rectas muestra la distancia desde el borde de café hacia los usos de suelo a la cual la broca no se dispersa.

El modelo uno tiene como estructura la ordenada del origen $(\alpha)$, la pendiente antes del punto de intersección de las dos rectas ( $\left.\beta_{-} 1\right)$, la pendiente después del punto de corte ( $\beta$ 2), y gamma es el punto de la intersección $(\gamma)$, en este caso la distancia. El modelo dos tiene la misma estructura del primero, lo que difiere es que se sustrae $\left(\beta \_2\right)$ en la ecuación, ya que en este caso solo interesa la pendiente $\left(\beta \_1\right)$ y su intersección con la segunda recta para encontrar el punto en la cual la broca no se dispersa.

Cuadro 3. Modelos generales mixtos para comparar la estimación de la relación foco de dispersión y la distancia en la que fueron colocadas las trampas.

Mixed General Models to compare the estimate of the dispersion focus ratio and the distance in which the traps were placed.

\begin{tabular}{|c|c|c|c|}
\hline \multirow{2}{*}{ Modelos } & \multicolumn{2}{|l|}{ Efecto fijo } & \multirow{2}{*}{ Efecto aleatorio } \\
\hline & Factor & Covariable & \\
\hline 1 & Tipo de uso+Distancia+Tipo de uso*Distancia & - & - \\
\hline 2 & Tipo de uso+Distancia+Tipo de uso*Distancia & P_café & - \\
\hline 3 & Tipo de uso+Distancia+Tipo de uso*Distancia & - & Sujeto \\
\hline 4 & Tipo de uso+Distancia+Tipo de uso*Distancia & P_café & Sujeto \\
\hline
\end{tabular}


Cuadro 4. Modelos no-lineales a dos tramos para comparar la estimación de la relación foco de dispersión y cada clase de distancia en la que fueron colocadas las trampas.

Non-linear models to two sections to compare the estimate of the dispersion focus ratio and each distance class in which the traps were placed.

\section{Modelos}

1

2

Donde: $\alpha$ : ordenada del origen, $\left(\beta_{1}\right)$ : la pendiente antes del punto de intersección de las dos rectas, $\beta_{2}$ : la pendiente después del punto de corte, $(\gamma)$ : punto de la intersección.

Finalmente se hicieron correlaciones de Pearson entre la variable respuesta con los predichos de los modelos que mejor ajustaron los datos. Esto con el fin de encontrar el mejor modelo entre los propuestos.

Dados los tipos de distribución espacial y las formas presentadas por las funciones empíricas conocidas se trabajaron con las distribuciones: exponencial y logarítmica. Se aplicaron técnicas de estadística clásica: a) análisis de regresión no lineal b) ajustes de distribuciones de los modelos propuestos c) comparación de las distribuciones de frecuencias empíricas con las distribuciones teórica propuestas. Para lo anterior se utilizó el software estadístico InfoStat versión 2011.

Ajustes no lineales para curvas de dispersión. Para cada uso de suelo, en cada una de las localidades, se calculó la distancia de dispersión de la broca. Para esto se despejó la distancia del modelo que mejor ajustó (Modelo 4) y luego se estimó esta igualando a cero la variable cantidad de broca. Debido a que el mejor modelo en todos los casos fue el cuatro (cuadro 1), para calcular la distancia se usó la función:

$$
X=\left(\frac{a-\ln (Y)}{b}\right)^{2}
$$

Donde

$X=$ distancia desde el borde del café

$a$ y $b=$ parámetros estimados de la función

$Y=$ cantidad de broca (número de capturas por punto de muestreo)

Para calcular la distancia a la cual la cantidad de broca es cero, y por ende ya no se dispersa, se iguala $Y$ a cero. Como el $\ln (Y)$ cuando $Y=0$ es 1, entonces la distancia a la que se dispersa la broca se calcula como:

$$
X=\left(\frac{(a-1)}{b}\right)^{2}
$$

Seguidamente los valores predichos por el modelo exponencial que mejor ajustó los datos versus las distancias se analizaron con todas las localidades.

\section{RESULTADOS}

Para la distancia de $10 \mathrm{~m}$ se observó una clara diferencia entre los usos caña y pasto con bosque; en la distancia $20 \mathrm{~m}$ el bosque ya presenta una dispersión inferior a caña y pasto. A los $30 \mathrm{~m}$ pasto es la que más dispersión presenta, continuando el orden pasto $>$ caña $>$ bosque hasta pasados los $80 \mathrm{~m}$, donde las diferencias entre los tres usos se diluyen (figura 2).

Ajuste de modelos lineales generales y mixtos para dispersión de broca. Al comparar los valores de AIC y BIC para las estructuras que se han ajustado, se encontró que el menor valor se obtiene con el Modelo 13 (AIC = 2.989,50; BIC = 3.376,13) (cuadro 5). Este modelo tiene como efecto fijo a tipo de uso, distancia y la interacción Tipo de uso×distancia, como efecto aleatorio a sujeto, con función de correlación corExp, con coordenada $\mathrm{X}$ a distancia y como criterio de agrupamiento a sujeto, se declara heteroscedasticidad varIdent con criterio de agrupamiento Tipo de uso $\times$ distancia.

El modelo general lineal y mixto que mejor ajustó los valores de predichos versus las distancias evidenció para la distancia de $10 \mathrm{~m}$ una clara diferencia entre los usos (dispersión en caña>pasto>bosque). A partir de los $30 \mathrm{~m}$ el bosque presenta la menor dispersión y la caña la mayor, pero también las diferencias se diluyen cerca de los 90 m (figura 3).

Posteriormente, se evaluaron los datos incluyendo transformaciones a logaritmo natural de la variable respuesta conteo, encontrando al Modelo 14 como el mejor, ya que posee los valores más bajos para los criterios de información AIC y BIC entre los modelos propuestos (cuadro 6).

Respecto a este análisis a pesar de haber interacción presente entre distancia y tipo de uso, esta se debe principalmente a la inclusión de la distancia 0 . Al no mostrarse esta distancia en el gráfico de predichos versus distancias no se ve una fuerte interacción. La tendencia general indica que el uso de suelo bosque es el que menos dispersión presenta (figura 4). 


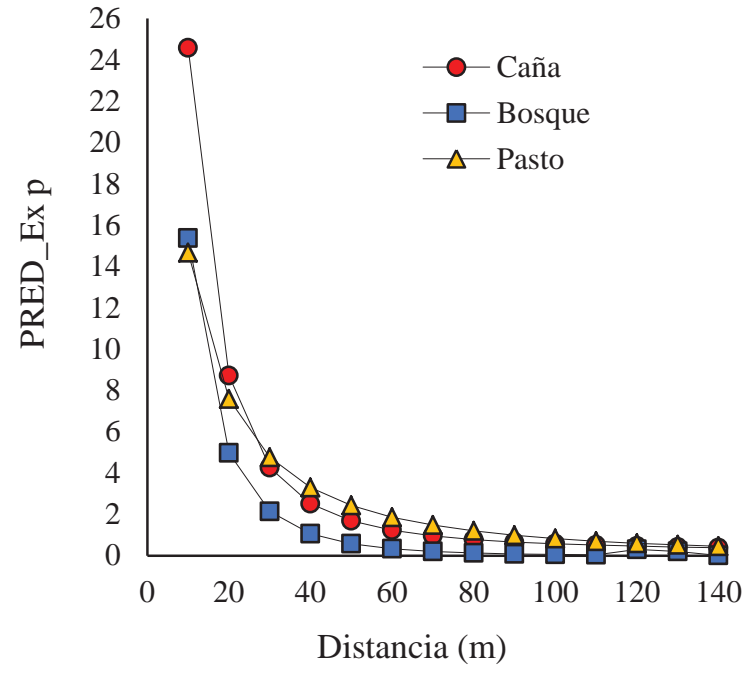

Figura 2. Valores predichos para la cantidad de broca capturada desde el borde del café hacia los usos de suelo adyacente vs las distancias, para el modelo exponencial.

Predicted values for the amount of coffee borer captured from the edge of the coffee to other land uses vs. the distances, for the exponential model.

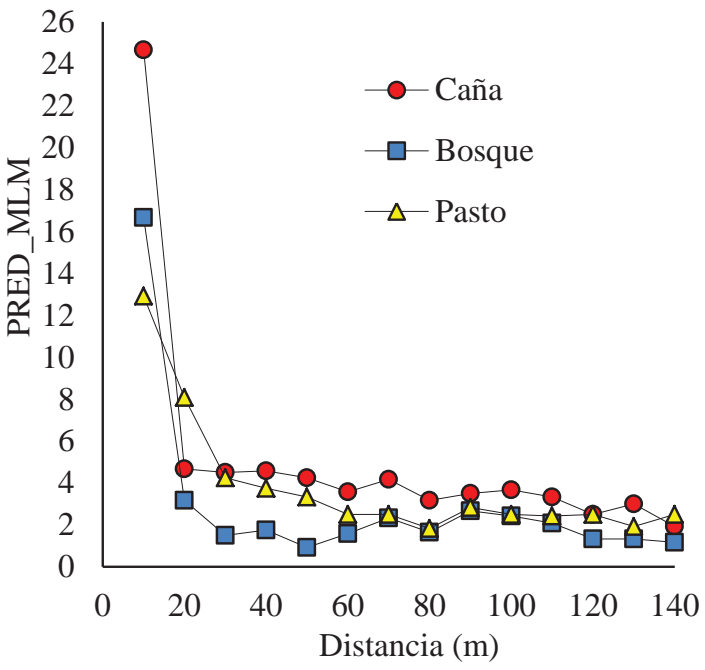

Figura 3. Valores predichos para la cantidad de broca capturada desde el borde del café hacia los usos de suelo adyacente vs las distancias, para el modelo lineal general mixto.

Predicted values for the amount of coffee borer captured from the edge of the coffee to other land uses vs. distances, for the mixed general linear model.

Cuadro 5. Modelos lineales generales y mixtos para comparar los criterios AIC y BIC y así contemplar cual fue el mejor modelo evaluado.

Models that best fit to compare the estimate of the dispersion focus ratio and each distance in which the traps were placed.

\begin{tabular}{|c|c|c|c|c|c|c|c|c|c|}
\hline Modelo & Cov & E.A & Corr & $\operatorname{coor} \mathrm{X}$ & Crit.Agrup & Heter & Crit.Agrup & AIC & BIC \\
\hline 1 & - & - & - & - & - & - & - & $6.200,77$ & $6.394,09$ \\
\hline 2 & - & Sujeto & - & - & - & - & - & $6.202,67$ & $6.400,18$ \\
\hline 3 & - & - & corExp & distancia & sujeto & - & - & $6.200,85$ & $6.398,37$ \\
\hline 4 & - & Sujeto & corExp & distancia & sujeto & - & - & $6.202,84$ & $6.404,56$ \\
\hline 5 & - & Sujeto & corGaus & distancia & sujeto & - & - & $6.202,92$ & $6.404,65$ \\
\hline 6 & - & Sujeto & corLin & distancia & sujeto & - & - & $6.202,93$ & $6.404,65$ \\
\hline 7 & - & Sujeto & corSpher & distancia & sujeto & - & - & $6.202,93$ & $6.404,65$ \\
\hline 8 & - & - & - & - & - & VarIdent & Var & $3.931,00$ & $4.128,52$ \\
\hline 9 & - & - & - & - & - & VarIdent & distancia & $3.282,90$ & $3.535,05$ \\
\hline 10 & - & - & - & - & - & VarExp & - & 3.828,99 & $4.026,51$ \\
\hline 11 & - & Sujeto & corExp & distancia & sujeto & VarIdent & distancia & $3.082,17$ & $3.342,73$ \\
\hline 12 & - & Sujeto & corExp & distancia & sujeto & varComb & Var3 & $3.178,83$ & 3.388,95 \\
\hline 13 & - & Sujeto & corExp & distancia & sujeto & varIdent & Tipo de uso*distancia & $2.989,50$ & $3.376,13$ \\
\hline 14 & - & Sujeto & corExp & distancia & sujeto & VarIdent & var3*tipo de uso & $3.107,43$ & $3.342,78$ \\
\hline 15 & - & - & corExp & distancia & sujeto & VarIdent & var3*tipo de uso & $3.129,46$ & $3.360,60$ \\
\hline 16 & P_cafe & Sujeto & corExp & distancia & sujeto & VarIdent & var3*tipo de uso & $3.122,42$ & $3.361,85$ \\
\hline
\end{tabular}


Cuadro 6. Modelos lineales generales y mixtos para comparar los criterios AIC y BIC y así contemplar cual fue el mejor modelo evaluado con la variable conteo transformada a logaritmo natural.

General and mixed linear models to compare the AIC and BIC criteria and thus to consider which was the best model evaluated with the variable counted transformed to natural logarithm.

\begin{tabular}{|c|c|c|c|c|c|c|c|c|c|}
\hline Modelo & Cov & E.A & Corr & $\operatorname{coor} \mathrm{X}$ & Crit.Agrup & Heter & Crit.Agrup & AIC & BIC \\
\hline 1 & - & - & - & - & - & - & - & $1.459,10$ & $1.652,42$ \\
\hline 2 & - & Sujeto & - & - & - & - & - & $1.311,44$ & $1.508,96$ \\
\hline 3 & - & - & corExp & distancia & sujeto & - & - & $1.304,32$ & $1.501,84$ \\
\hline 4 & - & Sujeto & corExp & distancia & sujeto & - & - & $1.275,80$ & $1.477,52$ \\
\hline 5 & - & Sujeto & corGaus & distancia & sujeto & - & - & $1.282,03$ & $1.483,75$ \\
\hline 6 & - & Sujeto & corLin & distancia & sujeto & - & - & $1.282,44$ & $1.484,16$ \\
\hline 7 & - & Sujeto & corSpher & distancia & sujeto & - & - & $1.282,44$ & $1.484,16$ \\
\hline 8 & - & - & - & - & - & VarIdent & Var & $1.438,72$ & $1.636,24$ \\
\hline 9 & - & - & - & - & - & VarIdent & distancia & $1.449,45$ & $1.701,61$ \\
\hline 10 & - & - & - & - & - & VarExp & - & $1.459,10$ & $1.652,42$ \\
\hline 11 & - & Sujeto & corExp & distancia & sujeto & VarIdent & distancia & $1.249,82$ & $1.510,37$ \\
\hline 12 & - & Sujeto & corExp & distancia & sujeto & varComb & Var3 & $1.233,80$ & $1.443,93$ \\
\hline 13 & - & Sujeto & corExp & distancia & sujeto & varIdent & Tipo de uso*distancia & $1.253,01$ & $1.639,64$ \\
\hline 14 & - & Sujeto & corExp & distancia & sujeto & VarIdent & var3*tipo de uso & $1.227,29$ & $1.462,63$ \\
\hline 15 & - & - & corExp & distancia & sujeto & VarIdent & var3*tipo de uso & $1.258,27$ & $1.489,41$ \\
\hline 16 & P_cafe & Sujeto & corExp & distancia & sujeto & VarIdent & var3*tipo de uso & $1.244,35$ & $1.483,78$ \\
\hline
\end{tabular}

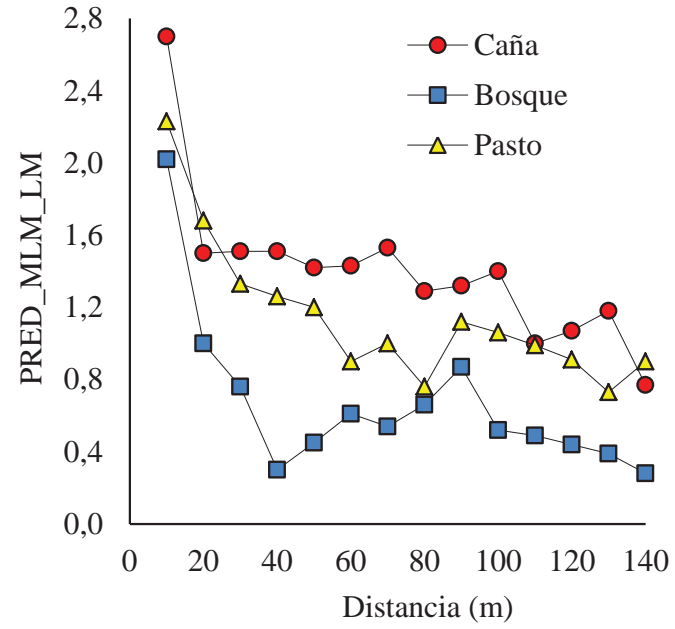

Figura 4. Valores predichos para la cantidad de broca capturada desde el borde del café hacia los usos de suelo adyacente vs las distancias, para el modelo lineal generalizado mixto con la variable respuesta transformada a logaritmo natural.

Predicted values for the amount of coffee borer captured from the edge of the coffee to other land uses vs. distances, for the mixed generalized linear model with the variable response transformed to natural logarithm.
Ajuste de modelos generalizados mixtos para dispersión de broca. Comparando los criterios AIC y BIC, se observó que el tercer modelo ajustado es el mejor, por lo tanto, la interpretación de las pruebas de hipótesis debe basarse en este último (cuadro 7). Este modelo tiene como estructura de covariable a P_café, como efectos fijos a tipo de uso, distancia y la interacción tipo de uso×distancia y como efecto aleatorio a sujeto.

La figura 5 muestra el análisis de interacción entre distancia y uso de suelo con base en los valores predichos por el modelo lineal generalizado mixto que mejor ajustó los datos versus las distancias usando el promedio sobre todas las repeticiones (una vez descontados los efectos de finca).

Ajuste de modelos de regresión por pasos (broken stick) para dispersión de broca. Estos modelos fueron analizados con diferentes estructuras para llegar al mejor ajuste. Sin embargo, se encontró que no existe un buen ajuste para estos, ya que los errores son muy grandes para cada uno de los parámetros y las estimaciones en su mayoría son negativas teniendo en cuenta cada uso de suelo y cada finca.

Comparación de los modelos alternativos que mejor se ajustaron. En este caso, los criterios AIC y BIC no se tu- 
Cuadro 7. Criterios de ajuste para la selección del mejor modelo lineal generalizado mixto.

Adjustment criteria for the selection of the best mixed generalized linear model.

\begin{tabular}{|c|c|c|c|c|c|c|}
\hline \multirow{2}{*}{ Modelos } & \multicolumn{2}{|l|}{ Efecto fijo } & \multirow{2}{*}{ Efecto aleatorio } & \multirow{2}{*}{ AIC } & \multirow{2}{*}{ BIC } & \multirow{2}{*}{ Desviance } \\
\hline & Factor & Covariable & & & & \\
\hline 1 & Tipo de uso + Distancia +Tipo de uso*Distancia & - & - & $1.5124,30$ & $15.317,30$ & $13.698,70$ \\
\hline 2 & Tipo de uso+Distancia+ Tipo de uso*Distancia & P_cafe & - & $1.2319,40$ & $12.516,70$ & $10.891,79$ \\
\hline 3 & Tipo de uso+Distancia+ Tipo de uso*Distancia & P_cafe & Sujeto & $3.498,13$ & $3.699,75$ & $3.404,13$ \\
\hline 4 & Tipo de uso+Distancia+ Tipo de uso*Distancia & - & Sujeto & $4.716,82$ & $4.914,15$ & $4.624,82$ \\
\hline
\end{tabular}

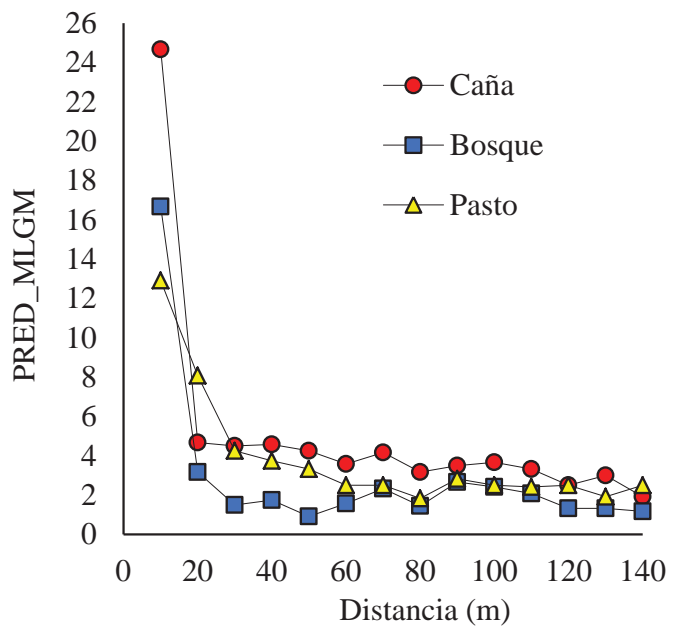

Figura 5. Valores predichos para la cantidad de broca capturada desde el borde del café hacia los usos de suelo adyacente vs las distancias, para el modelo lineal generalizado mixto.

Predicted values for the amount of coffee borer captured from the edge of the coffee to other land uses vs. distances, for the mixed generalized linear model.

vieron en cuenta para la comparación de los modelos evaluados, ya que estos tienen diferente variable respuesta. Es importante mencionar que, así como se encontraron diferencias en los modelos evaluados, también se encontraron similitudes. Para los mejores modelos analizados dentro de cada familia de modelos (cuadro 8), las distancias máximas de dispersión encontradas fueron en el uso de suelo caña, seguida por pasto y por último bosque.

Para los cuatro modelos las distancias de dispersión máximas se encontraron en el uso de suelo caña y las mínimas en el uso de suelo bosque. Siempre se encontraron diferencias entre el bosque vs caña y pasto; en cambio, para la comparación de medias de los usos de suelo adyacentes caña y pasto no hubo diferencias significativas entre estos usos, encontrando así en el uso de suelo bosque una mínima abundancia de broca y en caña y pasto una mayor abundancia del insecto.
Correlación de Pearson entre la variable respuesta conteo y los predichos de los modelos que mejor ajustaron los datos. El mejor modelo fue el lineal generalizado mixto, ya que el índice de correlación de Pearson entre la variable respuesta conteo y los predichos de este fue casi igual a uno $(r=0,99)$ (cuadro 9). Esto quiere decir que existe una correlación positiva mayor en comparación con los otros modelos.

Cuadro 8. Modelos que mejor se ajustaron para comparar la estimación de la relación foco de dispersión y cada distancia en la que fueron colocadas las trampas.

Models that best fit to compare the estimate of the dispersion focus ratio and each distance in which the traps were placed.

\begin{tabular}{cc}
\hline Modelo & Familia exponencial \\
\hline 4 & $Y=e^{(a-b \sqrt{x})}$ \\
Modelo & Modelos alternativos \\
3 & MLGM \\
13 & MLM \\
14 & MLM (variable respuesta transformada a ) \\
\hline
\end{tabular}

Cuadro 9. Correlación de Pearson entre la variable respuesta conteo y los predichos de los modelos que mejor ajustaron los datos.

Pearson's correlation between the variable response count and the predicted models that best fit the data.

\begin{tabular}{clcc}
\hline Modelo & \multicolumn{1}{c}{ Variable } & r de Pearson & Valor $P$ \\
\hline 4 & PRED_Exp & 0,87 & $<0,0001$ \\
13 & PRED_MLM & 0,58 & $<0,0001$ \\
14 & PRED_MLM_LN & 0,78 & $<0,0001$ \\
3 & PRED_MLGM & 0,99 & $<0,0001$ \\
\hline
\end{tabular}




\section{DISCUSIÓN}

Discusión estadística. En este estudio se probaron cinco modelos no-lineales propuestos por Kot et al. (1996) para evaluar la dispersión de organismos, los cuales permitieron explicar el movimiento de la broca del cafetal en los diferentes usos de suelo. El modelo que mejor se ajustó a los datos fue el de la familia exponencial negativa con raíz cuadrada de la distancia, el cual permite hallar diferencias entre los usos de suelo adyacente a los parches de café, así como también encontrar la distancia máxima aproximada en la que se puede dispersar el insecto.

Los resultados concuerdan con el estudio hecho por Wallace (1966), encontrando el mismo ajuste de curvas con el modelo exponencial negativo; no obstante, se observó diferencias con el estudio de Dobzhansky y Wright (1943) sobre genética de poblaciones naturales en Drosophila pseudoobscura, quienes afirman que la varianza de la dispersión incrementa linealmente con el tiempo.

Debido a la dificultad que presentan los modelos no lineales clásicos para encontrar una solución cuando el modelo es muy complejo, se optó por hacer un modelo para cada situación. Esto se traduce en una desventaja a la hora de generalizar los resultados.

Seguidamente se compararon tres modelos alternativos para poder ver la relación del tipo de cultivo y las distancias sin el sesgo dado por el efecto de finca dentro de cada localidad. Si bien la utilización de los modelos considerados permitió contemplar los factores asociados al muestreo de los datos, la potencia de cada uno fue diferente. Dentro de cada uno de los modelos usados, MLG, MLGM y MLG con variable transformada, distintas aproximaciones fueron evaluadas con los criterios de comparación de Akaike (1974) y de Schwartz (1978). Estos criterios permitieron seleccionar la mejor estrategia de análisis para cada tipo de modelo.

Se encontró que para el modelo lineal general y mixto con la variable respuesta conteo y el modelo lineal general mixto con la variable conteo transformada a Ln, no hizo falta la inclusión de la covariable, esto se debe a que no fue significativa y por ende no contribuye al ajuste de los datos. En este la cantidad de insectos en el café no estaba relacionada con los conteos dentro de los otros usos de suelo. Sin embargo, para los modelos lineales generalizados y mixtos se incluyó la covariable en el modelo siendo esta significativa. Esto quiere decir que la covariable si tiene una relación lineal con el conteo de insectos en los distintos usos cuando se usa esta aproximación.

En los modelos lineales generalizados mixtos se encontró una mayor abundancia de broca en la distancia 0 en el uso de suelo caña, por el contrario de los modelos lineales generales mixtos con la variable respuesta transformada a logaritmo natural se vio que en el uso de suelo bosque para la distancia 0 hay mayor abundancia de esta, esto se debe por la transformación, lo que significa que la media de la variable original no es igual a la media de los Ln (Y).
Por otra parte, al realizar los gráficos de los predichos de la cantidad de broca que se encuentra en el cafetal hacia los usos de suelo adyacentes vs las distancias, se encontró que para los cuatro modelos que mejor ajustaron los datos (uno dentro de cada gama de modelos), el patrón de dispersión de la broca es similar.

Cuando se quieren comparar diferentes modelos de una misma familia, estos deben ser para la misma variable respuesta (Mangeaud y Videla 2005). Dado que en este estudio se utilizaron transformaciones logarítmicas de la variable para incorporarla a los modelos generales lineales mixtos, resultaría inapropiado utilizar los métodos mencionados anteriormente.

Por esto, y también para comparar las otras dos familias de modelos (exp y MLGM), la comparación se realizó usando el coeficiente de correlación de Pearson entre la captura observada y la captura predicha por cada uno de los modelos, siendo el mejor aquel con un coeficiente mayor. En este caso fue el modelo lineal generalizado mixto, que permite ajustar directamente la variable número de insectos con una distribución Poisson, asumiendo entre otras cosas, que se incrementa la varianza al aumentar la media. Este modelo concuerda con el usado por (Mangeaud y Videla 2005) en su estudio de preferencia de alimentación en insectos, sugiriendo que para las variables de tipo conteo (con distribución Poisson) este modelo es más potente.

La ventaja de este modelo es que se utiliza para modelar recuentos que posean una distribución Poisson, en este caso con una función de enlace logarítmica. Bajo esta distribución podemos asegurarnos que los valores predichos serán positivos; una característica de esta distribución es que la varianza es igual a la media, haciendo que las varianzas aumenten cuando aumenta el conteo de insectos. La desventaja de este MLGM usando distribución Poisson con efectos aleatorios no siempre mantiene la relación media varianza, ya que la variabilidad añadida por los efectos aleatorios presentes puede dar lugar a una sobredispersión.

Excepto por el MLG, en todos los otros se encontró interacción entre distancia y uso de suelo. Sin embargo, esta interacción no se vio presente si consideramos desde la distancia 10 en adelante. Además, independientemente del tipo de uso, los conteos en el borde fueron muy superiores a los de las siguientes distancias. Por estos motivos los gráficos de dispersión de la broca por tipo de uso no incluyeron la distancia 0 .

El patrón de dispersión de $H$. hampei en el campo y la influencia del paisaje es un tema poco estudiado (Benavides et al. 2003), debido a ello, seguir los movimientos de los insectos bajo condiciones naturales es esencial para comprender su comportamiento (Hagler y Jackson 2001). Este tipo de estudios tienen una gran importancia debido a que la forma en que se dispersa una plaga en el agroecosistema es un indicativo, entre otros aspectos, de la capacidad de búsqueda de la plaga, de su capacidad para invadir nuevas áreas (Schuster et al. 1971) y del éxito reproductivo de las hembras (Weisser y Völkl 1997), características que 
pueden ayudar a definir estrategias para el control del insecto.

Con el modelo mejor ajustado, se encontró que la distancia máxima aproximada de dispersión alcanzada por la plaga, fue en el uso de suelo caña 31,2 m, lo cual concuerda con los resultados obtenidos por Castaño et al. (2005), quien encontró que la broca emergente del suelo después del zoqueo (poda) infestó frutos presentes en ramas distanciadas hasta $30 \mathrm{~m}$.

Las capturas de broca realizadas en las áreas de caña, bosque y pasto adyacentes al cafetal, son comparativamente bajas con las capturas en las áreas de café, lo cual refleja el comportamiento monófago de la broca cuya orientación del vuelo es hacia los compuestos volátiles generados por los frutos del café; sin embargo, Johanneson (1984) y Damon (2000) reportan una lista de 23 especies de 11 familias botánicas hospederas. Esto podría explicar el encontrar individuos en las áreas adyacentes al cafetal, especialmente en bosque, aunque esto no concuerda con Mathieu et al. (1999), quienes no encontraron capturas en las trampas ubicadas en un rango de $20 \mathrm{~m}$ de distancia al cafetal.

Las bajas capturas en áreas de bosque adyacentes a los cafetales encontrados en el estudio de Olivas (2010), permiten inferir que las áreas de bosque sirven como barrera para que la broca no se desplace, esto puede deberse a que el viento se lleva a la broca por encima del bosque, siendo este olfativamente repelente y por esto la broca no se acerca, en este caso las trampas con los difusores no actúan eficazmente ya que estas compiten con muchos olores. Sin embargo, para los usos de suelo caña y pasto se encontró una mayor capacidad del insecto para moverse. Una de las justificaciones a favor de esto es que los usos de suelo caña y pasto facilitan la dispersión al haber más corrientes de viento (Bustillo y Villalba 2007).

\section{CONCLUSIONES}

Estadísticas. Los modelos lineales generalizados mixtos presentan mejor ajuste para la variable respuesta del estudio de Olivas y son más potentes estadísticamente que los restantes modelos considerados.

Los modelos lineales mixtos son los más apropiados en el análisis de variables que poseen distribución normal (ln conteo), mientras que los modelos lineales generalizados mixtos son los más convenientes para aquellas con distribución Poisson (conteos).

Los resultados obtenidos refuerzan la importancia de probar diferentes modelos para estimar la dispersión de insectos y lograr mejores ajustes, con la consiguiente disminución de los errores.

Con el modelo lineal generalizado mixto, se puede estimar las distancias máximas de dispersión de la plaga.

Biológicas. Con el modelo antes mencionado, la distancia máxima aproximada de dispersión ocurre en el uso de suelo caña; esto indica que este uso de suelo adyacente sirve como tránsito para que la broca se mueva entre los cafetales.

De acuerdo a las curvas de dispersión obtenidas con el modelo ajustado, el uso de suelo que mejor controla el movimiento de la broca es el bosque, ya que las cantidades y distancias de captura fueron comparativamente menores que los usos pasto y caña. Por lo tanto, el bosque representa una barrera para el movimiento de la plaga entre los usos de suelo en donde hay parches de café, consecuentemente su implementación y/o protección se recomienda para disminuir la dispersión de broca.

Dados los resultados obtenidos en esta investigación se recalca la importancia de las configuraciones de paisaje para mitigar la dispersión de la broca.

\section{REFERENCIAS}

Akaike H. 1974. A new look at the statistical model identification. IEEE Transactions on Automatic Control 19(6): 716-723.

Baker P. 1984. Some aspects of the behavior of the coffee berry borer in relation to its control in southern Mexico (Coleoptera, Scolytidae). Folia Entomológica Mexicana 61: 9-24.

Benavides P, AE Bustillo, M Portilla, J Orozco. 2003. Classical biological control of coffee berry borer, Hypothenemus hampei (Coleoptera: Scolytidae) in Colombia with African parasitoids. In Proceedings, 1st International Symposium on Biological Control of Arthropods, Honolulu, Hawaii. p. 430-434.

Bustillo A, D Villalba. 2007. Efecto del clima y condiciones del cultivo en la biología y comportamiento de la broca del café, Hypothenemus hampei (Ferrari) Coleoptera: Curculionidae: Scolytinae. Manejo Da Broca-Do-Café: Workshop Internacional. 282 p.

Castaño A, P Benavides, PS Baker. 2005. Dispersión de Hypothenemus hampei en cafetales zoqueados. Cenicafé 56(2): 142-150.

Damon A. 2000. A review of the biology and control of the coffee berry borer, Hypothenemus hampei (Coleoptera: Scolytidae). Bulletin of entomological research 90(6): 453-465.

Dobzhansky T, S Wright. 1943. Genetics of natural populations. X. Dispersion rates in Drosophila pseudoobscura. Genetics 28(4): 304.

Dufour B. 2007. Condiciones de uso de las trampas en el control de la broca del café. In Manejo Da Broca-Do-Café. Workshop International. Londrina, Paraná, Brasil. p. 85-93.

Guharay F, J Monterrey, D Monterroso, C Staver. 2000. Manejo integrado de plagas en el cultivo de café. Serie Técnica, Manual Técnico. CATIE 44: 272.

Hagler JR, CG Jackson. 2001. Methods for marking insects: current techniques and future prospects. Annual review of entomology 46(1): 511-543.

Jaramillo JL, EC Montoya, P Benavides, CE Góngora. 2015. Beauveria bassiana y Metarhizium anisopliae para el control de broca del café en frutos del suelo. Revista Colombiana de Entomología 41(1): 95-105.

Johanneson, NE. 1984. Host pest relationship of the genus Hypothenemus (Scolytidae: Coleoptera) with special reference to the coffee berry borer, $H$. hampei. Journal of Coffee Research 14(2): 43-56. 
Kot M, MA Lewis, P Van Den Driessche. 1996. Dispersal data and the spread of invading organisms. Ecology 77(7): 20272042.

Mangeaud A, M Videla. 2005. En busca de la independencia perdida: la utilización de modelos lineales generalizados mixtos en pruebas de preferencia. Ecología austral 15(2): 199-206.

Mathieu F, LO Brun, B Frerot, DM Suckling, C Frampton. 1999. Progression in field infestation is linked with trapping of coffee berry borer, Hypothenemus hampei (Col., Scolytidae). Journal of Applied Entomology 123(9): 535-540.

Olivas AP. 2010. Efecto del uso del suelo adyacente al cafetal sobre la dispersión y dinámica poblacional de la broca $H y$ pothenemus hampei Ferrari y la abundancia de enemigos naturales en el cantón de Turrialba, Costa Rica. Tesis Magister Scientiae. Turrialba, Costa Rica. CATIE. 140 p.

Rebolledo W, ML Luis, AS Peña, I Rodríguez. 2016. Regulación biológica de Hypothenemus hampei (Ferrari) en café (Co- ffea arabica L.) con el uso de estractos vegetales en el municipio Junín, Táchira. Universidad\&Ciencia 5(2).

Rodríguez JR. 2014. Is it possible to achieve development on the basis of organic-coffee initiatives? The perspectives of a local-global business in Mayan Communities. Antipoda. Revista de Antropología y Arqueología 19: 217-241.

Schuster MF, JC Boling, JJ Marony Jr. 1971. Biological control of rhodesgrass scale by airplane releases of an introduced parasite of limited dispersing ability. In Huffaker CB ed. Biological Control. New York, United Estates. Springer. p. 227-250.

Schwarz G. 1978. Estimating the dimension of a model. The annals of statistics 6(2): 461-464.

Wallace B. 1966. On the dispersal of Drosophila. The American Naturalist 100(916): 551-563.

Weisser, WW; Völkl, W. 1997. Dispersal in the aphid parasitoid, Lysiphlebus cardui (Marshall) (Hym., Aphidiidae). Journal of Applied Entomology 121(1-5): 23-28.

Recibido: 13.06.17

Aceptado: 17.10.17 